University of South Florida

DIGITAL COMMONS

Digital Commons @ University of

@ UNIVERSITY OF SOUTH FLORIDA

South Florida

$1-1-2016$

\title{
2016 Work Plan USF System
}

USF

Follow this and additional works at: https://digitalcommons.usf.edu/usf_accountability_reports

\section{Scholar Commons Citation}

USF, "2016 Work Plan USF System" (2016). USF Accountability Reports. 63.

https://digitalcommons.usf.edu/usf_accountability_reports/63

This Article is brought to you for free and open access by the USF Archives at Digital Commons @ University of South Florida. It has been accepted for inclusion in USF Accountability Reports by an authorized administrator of Digital Commons @ University of South Florida. For more information, please contact digitalcommons@usf.edu. 


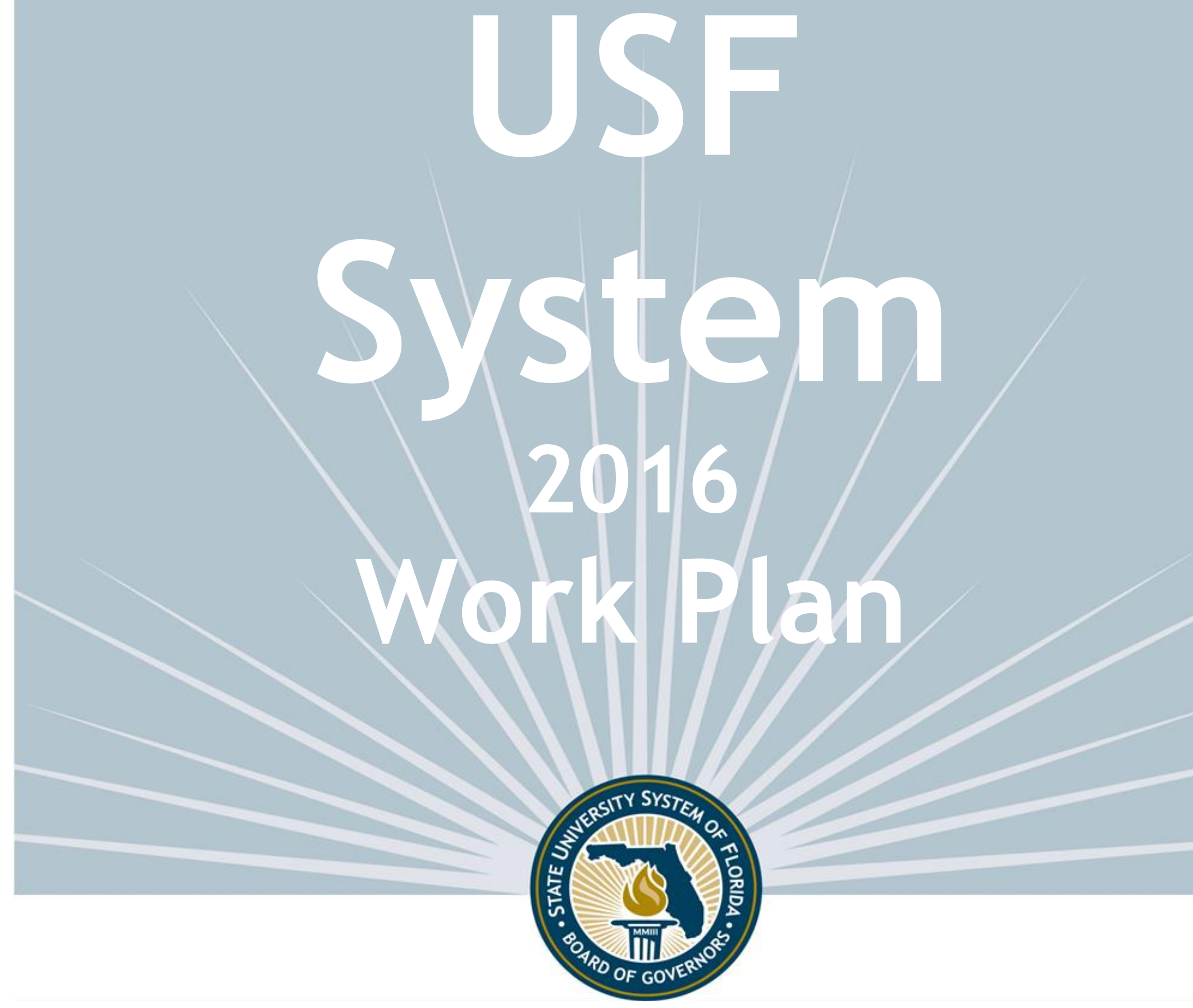

University of South Florida System

University Work Plan Presentation

for Board of Governors June 2016 Meeting

STATE UNIVERSITY SYSTEM of FLORIDA $\mid$ Board of Governors 
FINAL - JUNE 2016

\section{INTRODUCTION}

The State University System of Florida has developed three tools that aid in guiding the System's future.

1) The Board of Governors' 2025 System Strategic Plan is driven by goals and associated metrics that stake out where the System is headed;

2) The Board's Annual Accountability Report provides yearly tracking for how the System is progressing toward its goals;

3) Institutional Work Plans connect the two and create an opportunity for greater dialogue relative to how each institution contributes to the System's overall vision.

These three documents assist the Board with strategic planning and with setting short-, mid-and long-term goals. The Board will use these documents to help advocate for all System institutions and foster even greater coordination with the institutions and their Boards of Trustees.

Longer-term goals will inform future agendas of the Board's Strategic Planning Committee. The Board's acceptance of a work plan does not constitute approval of any particular component, nor does it supersede any necessary approval processes that may be required for each component. 


\section{TABLE OF CONTENTS}

1. STRATEGY

a. Mission Statement

b. Vision Statement

c. Statement of Strategy

d. Strengths and Opportunities

e. Key Initiatives \& Investments

\section{PERFORMANCE BASED FUNDING METRICS}

3. PREEMINENT RESEARCH UNIVERSITY METRICS

4. KEY PERFORMANCE INDICATORS

a. Teaching \& Learning

b. Scholarship, Research and Innovation

c. Institution Specific Goals

5. ENROLLMENT PLANNING

6. ACADEMIC PROGRAM COORDINATION

7. STUDENT DEBT \& NET COST

8. UNIVERSITY REVENUES

9. TUITION, FEES AND HOUSING PROJECTIONS

10. DEFINITIONS 


\section{MISSION STATEMENT (What is your purpose?)}

The University of South Florida System, which includes USF (in Tampa), USF St. Petersburg, and USF Sarasota-Manatee, catalyzes and coordinates initiatives that prepare students for successful 21st century careers; advances research, scholarship, and creative endeavors to improve the quality of life; and engages its communities across the Tampa Bay region for mutual benefits.

\section{VISION STATEMENT (What do you aspire to?)}

The University of South Florida System will empower and connect its institutions into a distinctive system that is nationally and globally recognized for innovation in teaching and research, for attracting outstanding and diverse scholars, staff and students, and for transforming the communities it serves.

\section{STATEMENT OF STRATEGY (How will you get there?)}

\section{Given your mission, vision, strengths and available resources, provide a brief description of your market and your strategy for addressing and leading it.}

The institutions of the USF System develop missions and strategic plans that best fit the communities they serve while also working together to achieve synergies and economies of scale. Under the leadership of the USF Board of Trustees the USF System embraces accountability, relying on a detailed dashboard to track key metrics such as graduation rates, retention rates, research support and faculty awards that are also key components of the Board of Governors' Strategic Plan, including performancebased funding metrics. Engaging in partnerships represents another important strategy; USF and USF St. Petersburg are both recognized by the Carnegie Foundation as community engaged universities. Achieving this recognition at USF Sarasota-Manatee is a key goal of its next strategic plan.

USF, in Tampa, is classified by Carnegie as a doctoral research university, highest research activity, attracting students and faculty of the highest caliber from across the world. The institution is working hard to position itself for AAU eligibility and preeminence as it maintains a commitment to student success, entrepreneurship and innovation, and global engagement.

USF St. Petersburg has developed a new strategic plan for 2014-19, which focuses on developing a distinctive identity as a valued member of the USF System, promoting faculty excellence, student success, strategic partnerships, and sustainable funding and infrastructure. The institution is now in the midst of a comprehensive implementation plan, working to bring those goals to life while maintaining positive momentum on key performance-funding metrics.

USF Sarasota-Manatee is also working toward goals as part of its strategic plan for 2015-2020. This plan focuses on enhancing student success, campus life, intentional enrollment management, high-quality teaching, community engagement and building a base of sustainable resources - all goals that will in turn help bolster the USF System's collective strengths. One such example of this is a burgeoning new partnership between USF and USF Sarasota-Manatee in engineering, where USF Sarasota-Manatee students will complete two years of pre-engineering course work on their home campus, complete an AA certificate and then transfer to the USF campus in Tampa to obtain a baccalaureate in engineering.

The USF System will seek out more of these types of programmatic partnerships among its separately accredited member institutions where it makes sense in order to serve workforce needs across the Tampa Bay region without unnecessary duplication. 


\section{STRENGTHS AND OPPORTUNITIES (within 3 years)}

\section{What are your core capabilities, opportunities and challenges for improvement?}

The core capabilities of the USF System represent the varied strengths of its three complementary member institutions. They include: high-impact scholarship; excellence in teaching and learning; an entrepreneurial spirit, partnerships; a focus on accountability and data-driven decision making; and community engagement. All three institutions are dedicated to student success, and students in the USF System benefit from having an array of course options across Tampa Bay. Programs hosted at one System institution are available to all USF System students.

The challenges for USF include maintaining momentum in student success and institutional quality with limited resources, as the university is working to increase budgetary efficiencies and hold down costs for students. Despite any challenges, the USF System is once again a top performer in the BOG's performance funding model and is looking forward to returning those new funds into key areas that will continue to enhance quality.

USF St. Petersburg and USF Sarasota-Manatee pride themselves on offering students an intimate campus experience and a high level of student-faculty interaction. At the same time, they benefit from associations, efficiencies of shared resources and opportunities for collaboration. Both are developing successful STEM programs that address local and statewide workforce needs and play an important role in regional economic development. At USFSP, challenges include growing needs for teaching and laboratory space as STEM programs prosper and enhancing student success to improve graduation and retention rates. For USFSM, the primary challenge is exploring ways to enhance the campus-life experience as it builds its lower-division student population. 


\section{KEY INITIATIVES \& INVESTMENTS (within 3 years)}

Describe your top three key initiatives for the next three years that will drive improvement in Academic Quality, Operational Efficiency, and Return on Investment.
1. Continue to enhance student success to maintain momentum as a top performer in the Board of Governors performance-funding model:
Each of the USF System institutions is working toward continuous improvement in graduation and retention rates, as well as connecting students with post-graduation employment opportunities. This goal is fueled by USF System's commitment to undergraduate research and its cultivation of a global curriculum; USFSP's focus on innovative retention strategies; and USFSM's efforts to enhance its campus environment and career prep services.

\section{Enhance academic program quality that prepares students for jobs:}

Students who graduate from the USF System should not only graduate on time, but also well prepared for leadership jobs in the workforce. Academic quality must go hand-in-hand with all of our student success initiatives. This is being accomplished through strategic hiring of high-quality, productive faculty; support for interdisciplinary programs that expose students to critical problems of today's world and innovative solutions; and development of partnerships across the USF System and in the larger Tampa Bay community - notably in STEM fields.

\section{Increase efficiencies and responsible financial practices:}

Across the USF System, financial resources are more precious than ever. As a continual top performer in the Board of Governors' performance-funding model, it is vital that we continue to put our investments to good use to further improve in those key metrics. The USF System is now revisiting its own shared services among its three member institutions, with the goal of increasing efficiencies and further streamlining business services. The USF System will also continue to practice transparent budgeting and maintain its commitment to keeping costs down for students as we maximize investments in areas that support their success. 


\section{PERFORMANCE BASED FUNDING METRICS}

\begin{tabular}{|c|c|c|c|c|c|c|}
\hline & $\begin{array}{c}2015 \\
\text { ACTUAL }\end{array}$ & $\begin{array}{c}2016 \\
\text { ACTUAL }\end{array}$ & $\begin{array}{c}2017 \\
\text { GOALS }\end{array}$ & $\begin{array}{l}2018 \\
\text { GOALS }\end{array}$ & $\begin{array}{c}2019 \\
\text { GOALS }\end{array}$ & $\begin{array}{l}2020 \\
\text { GOALS }\end{array}$ \\
\hline $\begin{array}{l}\text { Percent of Bachelor's Graduates } \\
\text { Enrolled or Employed }(\mathbf{\$ 2 5 , 0 0 0 + )} \\
\text { within the U.S. One Year After Graduation }\end{array}$ & $\begin{array}{l}65.3 \% \\
2012-13\end{array}$ & $\begin{array}{c}66.8 \% \\
2013-14\end{array}$ & $\begin{array}{c}66.8 \% \\
2014-15\end{array}$ & $\begin{array}{l}69.9 \% \\
2015-16\end{array}$ & $\begin{array}{l}71.9 \% \\
2016-17\end{array}$ & $\begin{array}{l}74.0 \% \\
2017-18\end{array}$ \\
\hline $\begin{array}{l}\text { Median Wages of Bachelor's } \\
\text { Graduates Employed Full-time } \\
\text { in Florida One-Year After Graduation }\end{array}$ & $\begin{array}{l}\$ 35,200 \\
2012-13\end{array}$ & $\begin{array}{l}\$ 36,300 \\
2013-14\end{array}$ & $\begin{array}{l}\$ 36,333 \\
2014-15\end{array}$ & $\begin{array}{c}\$ 36,767 \\
2015-16\end{array}$ & $\begin{array}{c}\$ 37,400 \\
2016-17\end{array}$ & $\begin{array}{c}\$ 37,933 \\
2017-18\end{array}$ \\
\hline $\begin{array}{l}\text { Cost per Bachelor's Degree } \\
\text { Costs to the University }\end{array}$ & $\begin{array}{c}\$ 25,490 \\
2010-14\end{array}$ & $\begin{array}{c}\$ 26,990 \\
2011-15\end{array}$ & $\begin{array}{l}\$ 26,990 \\
2012-16\end{array}$ & $\begin{array}{c}\$ 26,990 \\
2013-17\end{array}$ & $\begin{array}{c}\$ 26,990 \\
2014-18\end{array}$ & $\underset{2015-19}{\$ 26,990}$ \\
\hline $\begin{array}{l}\text { FTIC } 6 \text { year Graduation Rate * } \\
\text { for full- and part-time students }\end{array}$ & $\begin{array}{l}66.1 \% \\
2008-14\end{array}$ & $\begin{array}{l}67.8 \% \\
2009-15\end{array}$ & $\begin{array}{l}66.7 \% \\
2010-16\end{array}$ & $\begin{array}{l}69.6 \% \\
2011-17\end{array}$ & $\begin{array}{l}70.9 \% \\
2012-18\end{array}$ & $\begin{array}{l}73.5 \% \\
2013-19\end{array}$ \\
\hline $\begin{array}{l}\text { Academic Progress Rate } * \\
\text { FTIC } 2 \text { year Retention Rate with GPA>2 }\end{array}$ & $\begin{array}{l}85.3 \% \\
2013-14\end{array}$ & $\begin{array}{c}85.1 \% \\
2014-15\end{array}$ & $\begin{array}{l}85.6 \% \\
2015-16\end{array}$ & $\begin{array}{l}86.9 \% \\
2016-17\end{array}$ & $\begin{array}{l}87.9 \% \\
2017-18\end{array}$ & $\begin{array}{c}89.4 \% \\
2018-19\end{array}$ \\
\hline $\begin{array}{l}\text { Bachelor's Degrees Awarded } \\
\text { Within Programs of Strategic } \\
\text { Emphasis }\end{array}$ & $\begin{array}{l}51.0 \% \\
2013-14\end{array}$ & $\begin{array}{l}54.6 \% \\
2014-15\end{array}$ & $\begin{array}{l}54.8 \% \\
2015-16\end{array}$ & $\begin{array}{l}54.8 \% \\
2016-17\end{array}$ & $\begin{array}{c}54.9 \% \\
2017-18\end{array}$ & $\begin{array}{l}55.1 \% \\
2018-19\end{array}$ \\
\hline $\begin{array}{l}\text { University Access Rate } \\
\text { Percent of Fall Undergraduates } \\
\text { with a Pell grant }\end{array}$ & $\begin{array}{l}42.1 \% \\
\text { Fall } 2013\end{array}$ & $\begin{array}{l}41.6 \% \\
\text { Fall } 2014\end{array}$ & $\begin{array}{l}40.0 \% \\
\text { Fall } 2015\end{array}$ & $\begin{array}{l}40.1 \% \\
\text { Fall } 2016\end{array}$ & $\begin{array}{l}40.1 \% \\
\text { Fall } 2017\end{array}$ & $\begin{array}{l}40.1 \% \\
\text { Fall } 2018\end{array}$ \\
\hline $\begin{array}{l}\text { Graduate Degrees Awarded Within } \\
\text { Programs of Strategic Emphasis }\end{array}$ & $\begin{array}{l}69.0 \% \\
2013-14\end{array}$ & $\begin{array}{l}72.7 \% \\
2014-15\end{array}$ & $\begin{array}{l}74.0 \% \\
2015-16\end{array}$ & $\begin{array}{l}73.8 \% \\
2016-17\end{array}$ & $\begin{array}{l}73.6 \% \\
2017-18\end{array}$ & $\begin{array}{l}73.2 \% \\
2018-19\end{array}$ \\
\hline $\begin{array}{l}\text { BOG METRIC: } \\
\text { Percent of Bachelor's Degrees } \\
\text { Without Excess Hours }\end{array}$ & $\begin{array}{l}63.9 \% \\
2013-14\end{array}$ & $\begin{array}{c}65.8 \% \\
2014-15\end{array}$ & $\begin{array}{l}68.1 \% \\
2015-16\end{array}$ & $\begin{array}{l}70.6 \% \\
2016-17\end{array}$ & $\begin{array}{l}73.0 \% \\
2017-18\end{array}$ & $\begin{array}{l}75.4 \% \\
2018-19\end{array}$ \\
\hline $\begin{array}{l}\text { UBOT METRIC: } \\
\text { Number of Post-doctoral } \\
\text { Appointees }\end{array}$ & $\begin{array}{c}289 \\
\text { Fall } 2012\end{array}$ & $\begin{array}{l}321 \\
\text { Fall } 2013\end{array}$ & $\begin{array}{c}298 \\
\text { Fall } 2014\end{array}$ & $\begin{array}{c}282 \\
\text { Fall } 2015\end{array}$ & $\begin{array}{l}290 \\
\text { Fall } 2016\end{array}$ & $\begin{array}{l}297 \\
\text { Fall } 2017\end{array}$ \\
\hline
\end{tabular}

Note: Metrics are defined in appendix. For more information about the PBF model visit: http://www.flbog.edu/about/budget/performance_funding.php. *USF System data reflect the swirl among the USF System institutions (USF, USFSP, USFSM) 


\section{PREEMINENT RESEARCH UNIVERSITY FUNDING METRICS} USF-TAMPA CAMPUS ONLY

\begin{tabular}{|c|c|c|c|c|c|c|}
\hline & $\begin{array}{l}\text { BENCH- } \\
\text { MARKS }\end{array}$ & $\begin{array}{l}2016 \\
\text { ACTUAL }\end{array}$ & $\begin{array}{l}2017 \\
\text { GOALS }\end{array}$ & $\begin{array}{l}2018 \\
\text { GOALS }\end{array}$ & $\begin{array}{l}2019 \\
\text { GOALS }\end{array}$ & $\begin{array}{l}2020 \\
\text { GOALS }\end{array}$ \\
\hline $\begin{array}{l}\text { Average GPA and SAT Score } \\
\text { for incoming freshman in Fall semester }\end{array}$ & $\begin{array}{l}\text { 4.0 GPA } \\
1200 \mathrm{SAT}\end{array}$ & $\begin{array}{c}4.1 \\
1223 \\
\text { Fall } 2015\end{array}$ & $\begin{array}{c}4.0 \\
1220 \\
\text { Fall } 2016\end{array}$ & $\begin{array}{r}4.05 \\
1222 \\
\text { Fall } 2017\end{array}$ & $\begin{array}{l}4.075 \\
1224 \\
\text { Fall } 2018\end{array}$ & $\begin{array}{l}4.10 \\
1226 \\
\text { Fall } 2019\end{array}$ \\
\hline $\begin{array}{l}\text { Public University National Ranking } \\
\text { in more than one national ranking }\end{array}$ & Top 50 & $\begin{array}{c}4 \\
2016\end{array}$ & $\begin{array}{c}5 \\
2017\end{array}$ & $\begin{array}{c}5 \\
2018\end{array}$ & $\begin{array}{c}5 \\
2019\end{array}$ & $\begin{array}{c}5 \\
2020\end{array}$ \\
\hline $\begin{array}{l}\text { Freshman Retention Rate } \\
\text { Full-time, FTIC }\end{array}$ & $90 \%$ & $\begin{array}{l}88 \% \\
2014-15\end{array}$ & $\begin{array}{l}90 \% \\
2015-16\end{array}$ & $\begin{array}{r}91 \% \\
2016-17\end{array}$ & $\begin{array}{l}92 \% \\
2017-18\end{array}$ & $\begin{array}{l}93 \% \\
2018-19\end{array}$ \\
\hline $\begin{array}{l}\text { 6-year Graduation Rate } \\
\text { Full-time, FTIC }\end{array}$ & $70 \%$ & $\begin{array}{l}68 \% \\
2009-15\end{array}$ & $\begin{array}{c}66.5 \% \\
2010-16\end{array}$ & $\begin{array}{l}70.0 \% \\
2011-17\end{array}$ & $\begin{array}{l}72.0 \% \\
2012-18\end{array}$ & $\begin{array}{c}74.0 \% \\
2013-19\end{array}$ \\
\hline $\begin{array}{l}\text { National Academy } \\
\text { Memberships }\end{array}$ & 6 & $\begin{array}{c}8 \\
2016\end{array}$ & $\begin{array}{c}9 \\
2017\end{array}$ & $\begin{array}{l}10 \\
2018\end{array}$ & $\begin{array}{l}10 \\
2019\end{array}$ & $\begin{array}{l}10 \\
2020\end{array}$ \\
\hline $\begin{array}{l}\text { Science \& Engineering } \\
\text { Research Expenditures (\$M) }\end{array}$ & $\$ 200 M$ & $\begin{array}{l}\$ 420 \\
2014-15\end{array}$ & $\begin{array}{l}\$ 421 \\
2015-16\end{array}$ & $\begin{array}{l}\$ 427 \\
2016-17\end{array}$ & $\begin{array}{l}\$ 434 \\
2017-18\end{array}$ & $\begin{array}{l}\$ 440 \\
2018-19\end{array}$ \\
\hline $\begin{array}{l}\text { Non-Medical Science \& Engineering } \\
\text { Research Expenditures (\$M) }\end{array}$ & $\$ 150 M$ & $\$ 229$ & $\begin{array}{c}\$ 230 \\
2015-16\end{array}$ & $\begin{array}{l}\$ 233 \\
2016-17\end{array}$ & $\begin{array}{l}\$ 237 \\
2017-18\end{array}$ & $\begin{array}{l}\$ 241 \\
2018-19\end{array}$ \\
\hline $\begin{array}{l}\text { National Ranking in S.T.E.M. } \\
\text { Research Expenditures } \\
\text { includes public \& private institutions }\end{array}$ & $\begin{array}{l}\text { Top } 100 \\
\text { in } 5 \text { of } 8 \\
\text { disciplines }\end{array}$ & $\begin{array}{c}7 \\
2013-14\end{array}$ & $\begin{array}{c}7 \\
2014-15\end{array}$ & $\begin{array}{c}8 \\
2015-16\end{array}$ & $\begin{array}{c}8 \\
2016-17\end{array}$ & $\begin{array}{c}8 \\
2017-18\end{array}$ \\
\hline $\begin{array}{l}\text { Patents Awarded } \\
\text { over } 3 \text { year period }\end{array}$ & 100 & $\begin{array}{c}297 \\
2013-15\end{array}$ & $\begin{array}{c}291 \\
2014-16\end{array}$ & $\begin{array}{c}273 \\
2015-17\end{array}$ & $\begin{array}{c}276 \\
2016-18\end{array}$ & $\begin{array}{c}279 \\
2017-19\end{array}$ \\
\hline $\begin{array}{l}\text { Doctoral Degrees } \\
\text { Awarded Annually }\end{array}$ & 400 & $\begin{array}{c}601 \\
2014-15\end{array}$ & $\begin{array}{c}645 \\
2015-16\end{array}$ & $\begin{array}{c}650 \\
2016-17\end{array}$ & $\begin{array}{c}655 \\
2017-18\end{array}$ & $\begin{array}{c}660 \\
2018-19\end{array}$ \\
\hline $\begin{array}{l}\text { Number of Post-Doctoral } \\
\text { Appointees }\end{array}$ & 200 & $\begin{array}{c}289 \\
\text { Fall } 2012\end{array}$ & $\begin{array}{c}321 \\
\text { Fall } 2013\end{array}$ & $\begin{array}{c}298 \\
\text { Fall } 2014\end{array}$ & $\begin{array}{l}277 \\
\text { Fall } 2015\end{array}$ & $\begin{array}{c}285 \\
\text { Fall } 2016\end{array}$ \\
\hline Endowment Size (\$M) & $\$ 500 \mathrm{M}$ & $\begin{array}{l}\$ 417 \\
2014-15\end{array}$ & $\begin{array}{l}\$ 395 \\
2015-16\end{array}$ & $\begin{array}{l}\$ 412 \\
2016-17\end{array}$ & $\begin{array}{l}\$ 432 \\
2017-18\end{array}$ & $\begin{array}{l}\$ 448 \\
2018-19\end{array}$ \\
\hline NUMBER OF METRICS ABOVE THE & HMARK & 9 & 10 & 11 & 11 & 11 \\
\hline
\end{tabular}

Note: Metrics are defined in appendix. For more information about Preeminent state research universities, see 1001.7065 Florida Statutes. 


\section{KEY PERFORMANCE INDICATORS}

Teaching \& Learning Metrics (from 2025 System Strategic Plan that are not included in PBF or Preeminence)

\begin{tabular}{|c|c|c|c|c|c|c|}
\hline & $\begin{array}{c}2015 \\
\text { ACTUAL }\end{array}$ & $\begin{array}{c}2016 \\
\text { ACTUAL }\end{array}$ & $\begin{array}{c}2017 \\
\text { GOALS }\end{array}$ & $\begin{array}{c}2018 \\
\text { GOALS }\end{array}$ & $\begin{array}{c}2019 \\
\text { GOALS }\end{array}$ & $\begin{array}{c}2020 \\
\text { GOALS }\end{array}$ \\
\hline $\begin{array}{l}\text { 2. Freshmen in Top } 10 \% \\
\text { of Graduating High School Class }\end{array}$ & $\begin{array}{l}28.0 \% \\
\text { Fall } 2014\end{array}$ & $\begin{array}{l}30.0 \% \\
\text { Fall } 2015\end{array}$ & $\begin{array}{l}30.4 \% \\
\text { Fall } 2016\end{array}$ & $\begin{array}{l}30.6 \% \\
\text { Fall } 2017\end{array}$ & $\begin{array}{l}31.6 \% \\
\text { Fall } 2018\end{array}$ & $\begin{array}{c}31.9 \% \\
\text { Fall } 2019\end{array}$ \\
\hline $\begin{array}{l}\text { 3. Professional Licensure \& } \\
\text { Certification Exam Pass Rates } \\
\text { Above Benchmarks }\end{array}$ & $\begin{array}{l}3 \text { of } 5 \\
2013-14\end{array}$ & $\begin{array}{c}5 \text { of } 5 \\
2014-15\end{array}$ & 6 of 6 & $\begin{array}{c}6 \text { of } 6 \\
2016-17\end{array}$ & $\begin{array}{c}6 \text { of } 6 \\
2017-18\end{array}$ & 6 of 6 \\
\hline $\begin{array}{l}\text { 4. Time to Degree } \\
\text { Mean Years for FTICs } \\
\text { in } 120 \text { hr programs }\end{array}$ & $\begin{array}{c}4.7 \\
2013-14\end{array}$ & $\begin{array}{c}4.5 \\
2014-15\end{array}$ & $\begin{array}{c}4.5 \\
2015-16\end{array}$ & $\begin{array}{c}4.3 \\
2016-17\end{array}$ & $\begin{array}{c}4.3 \\
2017-18\end{array}$ & $\begin{array}{c}4.2 \\
2018-19\end{array}$ \\
\hline $\begin{array}{l}\text { 5. Four-Year FTIC } \\
\text { Graduation Rates } \\
\text { full- and part-time students }\end{array}$ & $\begin{array}{c}43.0 \% \\
2010-14\end{array}$ & $\begin{array}{c}48.0 \% \\
2011-15\end{array}$ & $\begin{array}{c}50.4 \% \\
2012-16\end{array}$ & $\begin{array}{c}53.4 \% \\
2013-17\end{array}$ & $\begin{array}{c}55.8 \% \\
2014-18\end{array}$ & $\begin{array}{c}57.9 \% \\
2015-19\end{array}$ \\
\hline $\begin{array}{l}\text { 8. Bachelor's Degrees Awarded } \\
\text { First Majors Only }\end{array}$ & $\begin{array}{c}9,390 \\
2013-14\end{array}$ & $\begin{array}{c}9,290 \\
2014-15\end{array}$ & $\begin{array}{c}9,081 \\
2015-16\end{array}$ & $\begin{array}{c}9,225 \\
2016-17\end{array}$ & $\begin{array}{c}9,358 \\
2017-18\end{array}$ & $\begin{array}{c}9,492 \\
2018-19\end{array}$ \\
\hline $\begin{array}{l}\text { 9. Graduate Degrees Awarded } \\
\text { First Majors Only }\end{array}$ & $\begin{array}{c}3,401 \\
2013-14\end{array}$ & $\begin{array}{c}3,773 \\
2014-15\end{array}$ & $\begin{array}{c}3,877 \\
2015-16\end{array}$ & $\begin{array}{c}3,954 \\
2016-17\end{array}$ & $\begin{array}{c}4,035 \\
2017-18\end{array}$ & $\begin{array}{c}4,092 \\
2018-19\end{array}$ \\
\hline $\begin{array}{l}\text { 10. Bachelor's Degrees Awarded } \\
\text { to African-American \& Hispanic } \\
\text { Students }\end{array}$ & $\begin{array}{c}27.6 \% \\
2013-14\end{array}$ & $\begin{array}{c}29.5 \% \\
2014-15\end{array}$ & $\begin{array}{c}29.6 \% \\
2015-16\end{array}$ & $\begin{array}{c}29.7 \% \\
2016-17\end{array}$ & $\begin{array}{c}30.0 \% \\
2017-18\end{array}$ & $\begin{array}{c}31.0 \% \\
2018-19\end{array}$ \\
\hline $\begin{array}{l}\text { 11. Adult (Aged } 25+\text { ) } \\
\text { Undergraduates Enrolled }\end{array}$ & $\begin{array}{l}24.0 \% \\
\text { Fall } 2013\end{array}$ & $\begin{array}{l}23.8 \% \\
\text { Fall } 2014\end{array}$ & $\begin{array}{l}22.9 \% \\
\text { Fall } 2015\end{array}$ & $\begin{array}{l}22.9 \% \\
\text { Fall } 2016\end{array}$ & $\begin{array}{l}21.8 \% \\
\text { Fall } 2017\end{array}$ & $\begin{array}{c}21.6 \% \\
\text { Fall } 2018\end{array}$ \\
\hline $\begin{array}{l}\text { 12. Percent of Undergraduate } \\
\text { FTE in Distance Learning } \\
\text { Courses }\end{array}$ & $\begin{array}{c}23.0 \% \\
2013-14\end{array}$ & $\begin{array}{c}26.0 \% \\
2014-15\end{array}$ & $\begin{array}{c}27.5 \% \\
2015-16\end{array}$ & $\begin{array}{c}28.2 \% \\
2016-17\end{array}$ & $\begin{array}{c}28.9 \% \\
2017-18\end{array}$ & $\begin{array}{c}29.6 \% \\
2018-19\end{array}$ \\
\hline $\begin{array}{l}\text { 16. Percent of Bachelor's } \\
\text { Degrees in STEM \& Health }\end{array}$ & $\begin{array}{c}34.0 \% \\
2013-14\end{array}$ & $\begin{array}{l}39.0 \% \\
2014-15\end{array}$ & $\begin{array}{c}40.5 \% \\
2015-16\end{array}$ & $\begin{array}{c}41.3 \% \\
2016-17\end{array}$ & $\begin{array}{c}42.5 \% \\
2017-18\end{array}$ & $\begin{array}{c}43.5 \% \\
2018-19\end{array}$ \\
\hline $\begin{array}{l}\text { 18. Percent of Graduate Degrees } \\
\text { in STEM \& Health }\end{array}$ & $\begin{array}{c}52.0 \% \\
2013-14\end{array}$ & $\begin{array}{l}57.0 \% \\
2014-15\end{array}$ & $\begin{array}{c}59.6 \% \\
2015-16\end{array}$ & $\begin{array}{c}60.5 \% \\
2016-17\end{array}$ & $\begin{array}{c}61.5 \% \\
2017-18\end{array}$ & $\begin{array}{c}62.5 \% \\
2018-19\end{array}$ \\
\hline IMPROVING METRICS & & $\begin{array}{c}10 \\
\text { of } 11\end{array}$ & $\begin{array}{c}\mathbf{9} \\
\text { of } 11\end{array}$ & $\begin{array}{c}9 \\
\text { of } 11\end{array}$ & $\begin{array}{c}9 \\
\text { of } 11\end{array}$ & $\begin{array}{c}10 \\
\text { of } 11\end{array}$ \\
\hline
\end{tabular}




\section{KEY PERFORMANCE INDICATORS (continued)}

Scholarship, Research and Innovation Metrics (from the 2025 System Strategic Plan)

\begin{tabular}{|c|c|c|c|c|c|c|}
\hline & $\begin{array}{c}2015 \\
\text { ACTUAL } \\
\end{array}$ & $\begin{array}{c}2016 \\
\text { ACTUAL }\end{array}$ & $\begin{array}{c}2017 \\
\text { GOALS }\end{array}$ & $\begin{array}{c}2018 \\
\text { GOALS }\end{array}$ & $\begin{array}{c}2019 \\
\text { GOALS }\end{array}$ & $\begin{array}{r}2020 \\
\text { GOALS }\end{array}$ \\
\hline 20. Faculty Awards & $\begin{array}{c}7 \\
2012\end{array}$ & $\begin{array}{c}8 \\
2013\end{array}$ & $\begin{array}{c}7 \\
2014\end{array}$ & $\begin{array}{c}8 \\
2015\end{array}$ & $\begin{array}{c}9 \\
2016\end{array}$ & $\begin{array}{l}10 \\
2017\end{array}$ \\
\hline $\begin{array}{l}\text { 22. Total Research } \\
\text { Expenditures (\$M) }\end{array}$ & $\begin{array}{r}\$ 497 \\
2013-14\end{array}$ & $\begin{array}{l}\$ 494 \\
2014-15\end{array}$ & $\begin{array}{l}\$ 495 \\
2015-16\end{array}$ & $\begin{array}{l}\$ 510 \\
2016-17\end{array}$ & $\begin{array}{l}\$ 525 \\
2017-18\end{array}$ & $\begin{array}{l}\$ 541 \\
2018-19\end{array}$ \\
\hline $\begin{array}{l}\text { 23. Research Expenditures } \\
\text { Funded from External Sources }\end{array}$ & $\begin{array}{l}60 \% \\
2013-14\end{array}$ & $\begin{array}{l}55 \% \\
2014-15\end{array}$ & $\begin{array}{l}56 \% \\
2015-16\end{array}$ & $\begin{array}{l}57 \% \\
2016-17\end{array}$ & $\begin{array}{l}58 \% \\
2017-18\end{array}$ & $\begin{array}{l}59 \% \\
2018-19\end{array}$ \\
\hline $\begin{array}{l}\text { 25. Licenses/Options } \\
\text { Executed }\end{array}$ & $\begin{array}{c}75 \\
2012-13\end{array}$ & $\begin{array}{c}91 \\
2013-14\end{array}$ & $\begin{array}{c}119 \\
2014-15\end{array}$ & $\begin{array}{c}120 \\
2015-16\end{array}$ & $\begin{array}{c}121 \\
2016-17\end{array}$ & $\begin{array}{c}122 \\
2017-18\end{array}$ \\
\hline $\begin{array}{l}\text { 26. Number of Start-up } \\
\text { Companies Created }\end{array}$ & $\begin{array}{c}11 \\
2013-14\end{array}$ & $\begin{array}{c}11 \\
2014-15\end{array}$ & $\begin{array}{c}8 \\
2015-16\end{array}$ & $\begin{array}{c}9 \\
2016-17\end{array}$ & $\begin{array}{c}10 \\
2017-18 \\
\end{array}$ & $\begin{array}{c}11 \\
2018-19 \\
\end{array}$ \\
\hline IMPROVING METRICS & & $\begin{array}{c}2 \\
\text { of } 5\end{array}$ & $\begin{array}{c}3 \\
\text { of } 5\end{array}$ & $\begin{array}{c}5 \\
\text { of } 5\end{array}$ & $\begin{array}{c}5 \\
\text { of } 5\end{array}$ & $\begin{array}{c}5 \\
\text { of } 5\end{array}$ \\
\hline
\end{tabular}

Institution Specific Goals (optional)

To further distinguish the university's distinctive mission, the university may choose to provide additional narrative and metric goals that are based on the university's own strategic plan. 
FINAL - JUNE 2016

\section{ENROLLMENT PLANNING}

Planned Headcount Enrollment by Student Type (for all students at all campuses)

\begin{tabular}{|c|c|c|c|c|c|c|c|}
\hline & $\begin{array}{c}\text { FALL } 2013 \\
\text { ACTUAL }\end{array}$ & $\begin{array}{c}\text { FALL } 2014 \\
\text { ACTUAL }\end{array}$ & $\begin{array}{c}\text { FALL } 2015 \\
\text { ACTUAL }\end{array}$ & $\begin{array}{l}\text { FALL } 2016 \\
\text { PLAN }\end{array}$ & $\begin{array}{c}\text { FALL } 2017 \\
\text { PLAN }\end{array}$ & $\begin{array}{l}\text { FALL } 2018 \\
\text { PLAN }\end{array}$ & $\begin{array}{l}\text { FALL } 2019 \\
\text { PLAN }\end{array}$ \\
\hline \multicolumn{8}{|l|}{ UNDERGRADUATE } \\
\hline FTIC & 17,214 & 17,376 & 17,703 & 17,887 & 18,119 & 18,379 & 18,497 \\
\hline AA Transfers ${ }^{1}$ & 11,056 & 10,737 & 10,603 & 10,559 & 10,724 & 10,916 & 11,134 \\
\hline Other ${ }^{2}$ & 7,738 & 7,693 & 7,683 & 7,934 & 7,909 & 7,879 & 7,776 \\
\hline Subtotal & 36,008 & 35,806 & 35,989 & 36,380 & 36,752 & 37,173 & 37,406 \\
\hline \multicolumn{8}{|l|}{ GRADUATE $^{3}$} \\
\hline Master's & 6,806 & 6,950 & 7,160 & 7,253 & 7,379 & 7,507 & 7,639 \\
\hline Research Doctoral & 2,294 & 2,226 & 2,229 & 2,232 & 2,235 & 2,238 & 2,241 \\
\hline Professional Doctoral & 1,220 & 1,379 & 1,309 & 1,239 & 1,241 & 1,244 & 1,246 \\
\hline Subtotal & 10,320 & 10,555 & 10,698 & 10,724 & 10,855 & 10,989 & 11,126 \\
\hline \multicolumn{8}{|l|}{ UNCLASSIFIED } \\
\hline H.S. Dual Enrolled & 46 & 16 & 25 & 41 & 57 & 80 & 113 \\
\hline Other ${ }^{4}$ & 1,941 & 2,201 & 2,272 & 2,282 & 2,333 & 2,385 & 2,438 \\
\hline Subtotal & 1,987 & 2,217 & 2,297 & 2,323 & 2,390 & 2,465 & 2,551 \\
\hline TOTAL & 48,315 & 48,578 & 48,984 & 49,426 & 49,997 & 50,628 & 51,083 \\
\hline
\end{tabular}

Notes: This table reports the number of students enrolled at the university by student type categories. The determination for undergraduate, graduate and unclassified is based on the institutional class level values. Unclassified refers to a student who has not yet been formally admitted into a degree program but is enrolled. The student type for undergraduates is based on the Type of Student at Time of Most Recent Admission. The student type for graduates is based on the degree that is sought and the student CIP code. (1) Includes AA Transfers from the Florida College System. (2) Undergraduate - Other includes Post-Baccalaureates who are seeking a degree. (3) Includes Medical students. (4) Unclassified - Other includes Post-Baccalaureates who are not seeking a degree.

Planned FTE Enrollment by Method of Instruction (for all students at all campuses)

\begin{tabular}{|c|c|c|c|c|c|c|c|}
\hline & $\begin{array}{l}\text { 2012-13 } \\
\text { ACTUAL }\end{array}$ & $\begin{array}{l}2013-14 \\
\text { ACTUAL }\end{array}$ & $\begin{array}{l}2014-15 \\
\text { ACTUAL }\end{array}$ & $\begin{array}{c}2015-16 \\
\text { PLAN }\end{array}$ & $\begin{array}{l}2016-17 \\
\text { PLAN }\end{array}$ & $\begin{array}{l}2017-18 \\
\text { PLAN }\end{array}$ & $\begin{array}{c}2018-19 \\
\text { PLAN }\end{array}$ \\
\hline \multicolumn{8}{|l|}{ UNDERGRADUATE } \\
\hline Distance (80-100\%) & 7,804 & 7,867 & 8,745 & 9,413 & 9,725 & 10,047 & 10,381 \\
\hline Hybrid (50-79\%) & 670 & 580 & 522 & 235 & 237 & 240 & 242 \\
\hline Traditional (0-50\%) & 25,807 & 25,396 & 24,414 & 24,520 & 24,510 & 24,476 & 24,441 \\
\hline Subtotal & 34,281 & 33,843 & 33,680 & 34,168 & 34,472 & 34,763 & 35,064 \\
\hline \multicolumn{8}{|l|}{ GRADUATE } \\
\hline Distance (80-100\%) & 1,747 & 1,825 & 2,051 & 2,105 & 2,171 & 2,240 & 2,310 \\
\hline Hybrid (50-79\%) & 215 & 233 & 204 & 68 & 69 & 69 & 70 \\
\hline Traditional (0-50\%) & 6,006 & 6,223 & 6,301 & 6,504 & 6,613 & 6,718 & 6,825 \\
\hline Subtotal & 7,968 & 8,282 & 8,555 & 8,677 & 8,853 & 9,027 & 9,205 \\
\hline
\end{tabular}

Note: Full-time Equivalent (FTE) student is a measure of instructional activity that is based on the number of credit hours that students enroll. FTE is based on the standard national definition, which divides undergraduate credit hours by 30 and graduate credit hours by 24 . Distance Learning is a course in which at least 80 percent of the direct instruction of the course is delivered using some form of technology when the student and instructor are separated by time or space, or both (per 1009.24(17), F.S.). Hybrid is a course where $50 \%$ to $79 \%$ of the instruction is delivered using some form of technology, when the student and instructor are separated by time or space, or both (per SUDS data element 2052). Traditional refers to primarily face to face instruction utilizing some form of technology for delivery of supplemental course materials for no more than $49 \%$ of instruction (per SUDS data element 2052). 


\section{ENROLLMENT PLANNING (continued)}

\section{Planned FTE Enrollment Plan by Student Level}

\begin{tabular}{|c|c|c|c|c|c|c|c|c|c|}
\hline & $\begin{array}{l}2014-15 \\
\text { ACTUAL } \\
\end{array}$ & $\begin{array}{c}2015-16 \\
\text { ESTIMATE }\end{array}$ & $\begin{array}{c}2016-17 \\
\text { PLAN }\end{array}$ & $\begin{array}{c}2017-18 \\
\text { PLAN }\end{array}$ & $\begin{array}{c}2018-19 \\
\text { PLAN }\end{array}$ & $\begin{array}{c}2019-20 \\
\text { PLAN }\end{array}$ & $\begin{array}{c}2020-21 \\
\text { PLAN } \\
\end{array}$ & $\begin{array}{c}2021-22 \\
\text { PLAN } \\
\end{array}$ & $\begin{array}{c}\text { Annual } \\
\text { Growth } \\
\text { Rate* }^{*}\end{array}$ \\
\hline \multicolumn{10}{|c|}{ STATE FUNDABLE } \\
\hline \multicolumn{10}{|l|}{ RESIDENT } \\
\hline LOWER & 11,625 & 11,724 & 11,824 & 11,926 & 12,032 & 12,141 & 12,253 & 12,368 & $0.9 \%$ \\
\hline UPPER & 18,632 & 18,496 & 18,665 & 18,839 & 19,020 & 19,206 & 19,400 & 19,600 & $1.0 \%$ \\
\hline GRAD I & 4,505 & 4,371 & 4,462 & 4,556 & 4,652 & 4,750 & 4,851 & 4,954 & $2.1 \%$ \\
\hline GRAD II & 1,258 & 1,155 & 1,175 & 1,196 & 1,218 & 1,240 & 1,262 & 1,285 & $1.8 \%$ \\
\hline $\begin{array}{c}\text { TOTAL } \\
\text { NON RESID }\end{array}$ & 36,019 & 35,745 & 36,126 & 36,518 & 36,922 & 37,337 & 37,766 & 38,208 & $1.1 \%$ \\
\hline LOWER & 1,181 & 1,424 & 1,431 & 1,438 & 1,444 & 1,451 & 1,459 & 1,466 & $0.5 \%$ \\
\hline UPPER & 1,175 & 1,460 & 1,468 & 1,477 & 1,486 & 1,495 & 1,504 & 1,514 & $0.6 \%$ \\
\hline GRAD I & 1,226 & 1,473 & 1,501 & 1,529 & 1,557 & 1,586 & 1,615 & 1,645 & $1.9 \%$ \\
\hline GRAD II & 877 & 926 & 942 & 959 & 976 & 994 & 1,012 & 1,030 & $1.8 \%$ \\
\hline TOTAL & 4,459 & 5,283 & 5,342 & 5,402 & 5,463 & 5,526 & 5,590 & 5,655 & $1.1 \%$ \\
\hline \multicolumn{10}{|l|}{ TOTAL } \\
\hline LOWER & 12,806 & 13,149 & 13,268 & 13,390 & 13,506 & 13,592 & 13,711 & 13,834 & $0.8 \%$ \\
\hline UPPER & 19,806 & 19,956 & 20,157 & 20,341 & 20,529 & 20,701 & 20,904 & 21,114 & $0.9 \%$ \\
\hline GRAD I & 5,733 & 5,845 & 5,964 & 6,086 & 6,210 & 6,337 & 6,468 & 6,601 & $2.0 \%$ \\
\hline GRAD II & 2,133 & 2,079 & 2,116 & 2,154 & 2,193 & 2,232 & 2,272 & 2,313 & $1.8 \%$ \\
\hline TOTAL & 40,478 & 41,029 & 41,505 & 41,971 & 42,438 & 42,863 & 43,356 & 43,863 & $1.1 \%$ \\
\hline \multicolumn{10}{|c|}{ NOT STATE FUNDABLE } \\
\hline LOWER & 613 & 631 & 634 & 616 & 609 & 602 & 595 & 588 & $-1.5 \%$ \\
\hline UPPER & 455 & 433 & 422 & 426 & 429 & 433 & 438 & 441 & $0.9 \%$ \\
\hline GRAD I & 665 & 693 & 705 & 717 & 731 & 745 & 758 & 772 & $1.8 \%$ \\
\hline GRAD II & 24 & 58 & 59 & 60 & 61 & 62 & 63 & 65 & $2.0 \%$ \\
\hline TOTAL & 1,757 & 1,815 & 1,820 & 1,818 & 1,830 & 1,843 & 1,854 & 1,866 & $0.5 \%$ \\
\hline
\end{tabular}

Note: Full-time Equivalent (FTE) student is a measure of instructional activity that is based on the number of credit hours that students enroll. FTE is based on the standard national definition, which divides undergraduate credit hours by 30 and graduate credit hours by 24 . Note*: The Planned Annual Growth Rate is a compounded rate based on the following formula: (2021-22 value divided by the 2016-17 value) to the (1/5) exponent minus one.

Medical Student Headcount Enrollments

\begin{tabular}{lccccccccc} 
& $\begin{array}{c}2014-15 \\
\text { ACTUAL }\end{array}$ & $\begin{array}{c}2015-16 \\
\text { ESTIMATE }\end{array}$ & $\begin{array}{c}2016-17 \\
\text { PLAN }\end{array}$ & $\begin{array}{c}2017-18 \\
\text { PLAN }\end{array}$ & $\begin{array}{c}2018-19 \\
\text { PLAN }\end{array}$ & $\begin{array}{c}2019-20 \\
\text { PLAN }\end{array}$ & $\begin{array}{c}2020-21 \\
\text { PLAN }\end{array}$ & $\begin{array}{c}2021-22 \\
\text { PLAN }\end{array}$ & $\begin{array}{c}\text { Annual } \\
\text { Growth } \\
\text { Rate* }\end{array}$ \\
\hline MEDICAL DOCTORATES & & & & & & & & & \\
\hline RESIDENT & 438 & 430 & 415 & 415 & 413 & 406 & 406 & 406 & $-0.4 \%$ \\
NON-RESIDENT & 53 & 74 & 74 & 74 & 74 & 74 & 74 & 74 & $0.0 \%$ \\
\hline TOTAL & 491 & 504 & 489 & 489 & 487 & 480 & 480 & 480 & $-0.4 \%$
\end{tabular}


FINAL - JUNE 2016

\section{ACADEMIC PROGRAM COORDINATION}

New Programs For Consideration by University in AY 2016-17

The S.U.S. Council of Academic Vice Presidents (CAVP) Academic Program Coordination Work Group will review these programs as part of their on-going coordination efforts. The programs listed below are based on the 2015 Work Plan list for programs under consideration for 2016-17.

\begin{tabular}{|c|c|c|c|c|c|c|}
\hline PROGRAM TITLES & $\begin{array}{l}\text { CIP CODE } \\
\text { 6-digit }\end{array}$ & $\begin{array}{l}\text { AREA OF } \\
\text { STRATEGIC } \\
\text { EMPHASIS }\end{array}$ & $\begin{array}{c}\text { OTHER } \\
\text { UNIVERSITIES } \\
\text { WITH SAME } \\
\text { PROGRAM }\end{array}$ & $\begin{array}{l}\text { OFFERED VIA } \\
\text { DISTANCE } \\
\text { LEARNING } \\
\text { IN SYSTEM }\end{array}$ & $\begin{array}{l}\text { PROJECTED } \\
\text { ENROLLMENT } \\
\text { in 5th year }\end{array}$ & $\begin{array}{l}\text { PROPOSED } \\
\text { DATE OF } \\
\text { SUBMISSION } \\
\text { TO UBOT }\end{array}$ \\
\hline \multicolumn{7}{|l|}{ BACHELOR'S PROGRAMS } \\
\hline $\begin{array}{l}\text { BS Public Relations, Advertising, } \\
\text { And Applied Communications (USF) }\end{array}$ & 09.0900 & GAP & FSU & $25 \%$ & 580 & Fall 2016 \\
\hline
\end{tabular}

\section{MASTER'S, SPECIALIST AND OTHER ADVANCED MASTER'S PROGRAMS}

\begin{tabular}{|c|c|c|c|c|c|c|}
\hline $\begin{array}{l}\text { MS Public Relations, Advertising, } \\
\text { And Applied Communications (USF) }\end{array}$ & 09.0900 & GAP & FSU, UNF & $0 \%$ & 68 & Spring 2017 \\
\hline MS Conservation Biology (USFSP) & 26.1307 & STEM & UCF & $0 \%$ & 30 & Spring 2017 \\
\hline
\end{tabular}

DOCTORAL PROGRAMS

New Programs For Consideration by University in 2017-19

These programs will be used in the 2017 Work Plan list for programs under consideration for 2017-18.

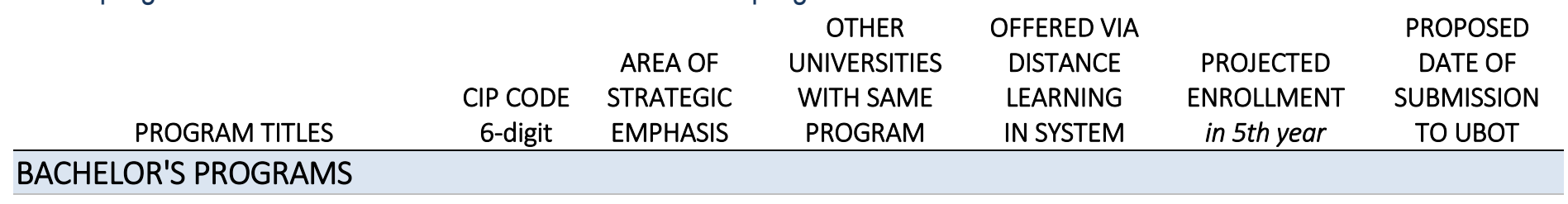

MASTER'S, SPECIALIST AND OTHER ADVANCED MASTER'S PROGRAMS

DOCTORAL PROGRAMS

\begin{tabular}{lcccccc}
\hline OTD Occupational Therapy (USF) & 51.2306 & HEALTH & - & $0 \%$ & 80 & TBD \\
\hline PhD Pharmacy (USF) & 51.2099 & STEM & FAMU, UF & $30 \%$ & 5 & TBD \\
\hline
\end{tabular}




\section{STUDENT DEBT \& NET COST}

\section{Student Debt Summary}

\begin{tabular}{lccccc} 
& $2010-11$ & $2011-12$ & $2012-13$ & $2013-14$ & $2014-15$ \\
\hline Percent of Bachelor's Recipients with Debt & $53 \%$ & $57 \%$ & $59 \%$ & $59 \%$ & $59 \%$ \\
\hline $\begin{array}{l}\text { Average Amount of Debt } \\
\text { for Bachelor's who have graduated with debt }\end{array}$ & $\$ 21,780$ & $\$ 22,620$ & $\$ 22,720$ & $\$ 22,610$ & $\$ 22,650$ \\
& & & & & $2012-15$ \\
NSLDS Cohort Year & $2008-11$ & $2009-12$ & $2010-13$ & $2011-14$ & Preliminary \\
\hline Student Loan Cohort Default Rate (3rd Year) & $10.1 \%$ & $9.8 \%$ & $7.5 \%$ & $5.4 \%$ & $5.2 \%$
\end{tabular}

Cost of Attendance (for Full-Time Undergraduate Florida Residents in the Fall and Spring of 2015-16)

\section{See Individual USF Institution Work Plans}

Estimated Net Cost by Family Income (for Full-Time Undergraduate Florida Residents in the Fall and Spring of 2015-16)

\begin{tabular}{|c|c|c|c|c|c|c|}
\hline \multirow{2}{*}{$\begin{array}{l}\text { FAMILY } \\
\text { INCOME } \\
\text { GROUPS }\end{array}$} & \multicolumn{2}{|c|}{$\begin{array}{l}\text { FULL-TIME RESIDENT } \\
\text { UNDFRGRADUATFS }\end{array}$} & \multirow{2}{*}{$\begin{array}{c}\text { AVG. NET } \\
\text { COST OF } \\
\text { ATTENDANCE }\end{array}$} & \multirow{2}{*}{$\begin{array}{l}\text { AVG. NET } \\
\text { TUITION } \\
\& \text { FEES }\end{array}$} & \multirow{2}{*}{$\begin{array}{c}\text { AVG. } \\
\text { GIFT AID } \\
\text { AMOUNT }\end{array}$} & \multirow{2}{*}{$\begin{array}{c}\text { AVG. } \\
\text { LOAN } \\
\text { AMOUNT }\end{array}$} \\
\hline & HEADCOUNT & PERCENT & & & & \\
\hline Below $\$ 40,000$ & 6,482 & $33 \%$ & $\$ 11,308$ & $-\$ 3,050$ & $\$ 8,832$ & $\$ 4,012$ \\
\hline$\$ 40,000-\$ 59,999$ & 2,313 & $12 \%$ & $\$ 13,275$ & $-\$ 968$ & $\$ 6,779$ & $\$ 3,760$ \\
\hline$\$ 60,000-\$ 79,999$ & 1,769 & $9 \%$ & $\$ 15,448$ & $\$ 1,416$ & $\$ 4,507$ & $\$ 4,458$ \\
\hline$\$ 80,000-\$ 99,999$ & 1,503 & $8 \%$ & $\$ 16,477$ & $\$ 2,588$ & $\$ 3,364$ & $\$ 4,781$ \\
\hline$\$ 100,000$ Above & 4,413 & $22 \%$ & $\$ 16,626$ & $\$ 2,591$ & $\$ 3,392$ & $\$ 3,943$ \\
\hline Not Reported & 3,103 & $16 \%$ & $\mathrm{n} / \mathrm{a}$ & $\$ 3,398$ & $\$ 2,515$ & $\$ 134$ \\
\hline TOTAL & 19,583 & $100 \%$ & $\$ 13,925^{*}$ & $\$ 328$ & $\$ 5,552$ & $\$ 3,452$ \\
\hline
\end{tabular}

Notes: This data only represents Fall and Spring financial aid data and is accurate as of March 31, 2016. Please note that small changes to Spring 2016 awards are possible before the data is finalized. Family Income Groups are based on the Total Family Income (including untaxed income) as reported on student FAFSA records. Full-time Students is a headcount based on at least 24 credit hours during Fall and Spring terms. Average Gift Aid includes all grants and scholarships from Federal, State, University and other private sources administered by the Financial Aid Office. Student waivers are also included in the Gift Aid amount. Gift Aid does not include the parental contribution towards EFC. Net Cost of Attendance is the actual average of the total Costs of Attendance (which will vary by income group due to the diversity of students living on- \& off- campus) minus the average Gift Aid amount. Net Tuition \& Fees is the actual average of the total costs of tuition and fees (which will vary by income group due to the amount of credit hours students are enrolled) minus the average Gift Aid amount (see page 16 for list of fees that are included). Average Loan Amount includes Federal (Perkins, Stafford, Ford Direct, and PLUS loans) and all private loans. 'Not Reported' represents the students who did not file a FAFSA. The bottom-line Total/Average represents the average of all full-time undergraduate Florida residents (note*: the total Net Cost of Attendance does not include students who did not report their family income data. 


\section{UNIVERSITY REVENUES}

University Revenues (in Millions of Dollars)

EDUCATION \& GENERAL

\begin{tabular}{c}
\hline Main Operations \\
State Funds \\
Tuition
\end{tabular}

Subtotal
2014-15

$\$ 278.2$

$\$ 193.1$

$\$ 471.2$
2015-16

$\$ 293.6$

$\$ 200.3$

$\$ 493.9$

Health-Science Center / Medical Schools

State Funds

$\$ 74.7$

$\$ 74.2$

Tuition

$\$ 50.6$

$\$ 57.7$

SUBTOTAL

$\$ 125.2$

$\$ 131.9$

E\&G TOTAL

$\$ 596.5$

$\$ 625.7$

\section{OTHER BUDGET ENTITIES}

$\begin{array}{lcc}\text { Auxiliary Enterprises } & \$ 204.2 & \$ 246.1 \\ \text { Contracts \& Grants } & \$ 281.3 & \$ 389.2 \\ \text { Local Funds } & \$ 435.0 & \$ 482.7 \\ \text { Faculty Practice Plans } & \$ 229.2 & \$ 245.9\end{array}$

Note: State funds include recurring and non-recurring General Revenue funds, Lottery funds appropriated by the Florida Legislature. Actual tuition includes base tuition and tuition differential fee revenues for resident and nonresident undergraduate and graduate students net of waivers. Source: Tables $1 \mathrm{~A} \& 1 \mathrm{E}$ of the annual Accountability Report. 


\title{
UNIVERSITY TUITION, FEES AND HOUSING PROJECTIONS
}

\author{
See Individual USF Institution Work Plans
}




\section{DEFINITIONS}

\section{Performance Based Funding}

Percent of Bachelor's

Graduates Enrolled or

Employed $(\$ 25,000+)$

in the U.S. One Year After

Graduation
This metric is based on the percentage of a graduating class of bachelor's degree recipients who are enrolled or employed (earning at least $\$ 25,000$ ) somewhere in the United States. Students who do not have valid social security numbers and are not found enrolled are excluded. Note: This data now non-Florida employment data.

Sources: State University Database System (SUDS), Florida Education \& Training Placement Information Program (FETPIP) analysis of Wage Record Interchange System (WRIS2) and Federal Employment Data Exchange (FEDES), and National Student Clearinghouse (NSC).

\section{Median Wages \\ of Bachelor's Graduates Employed Full-time in Florida One Year After Graduation}

This metric is based on annualized Unemployment Insurance (UI) wage data from the fourth fiscal quarter after graduation for bachelor's recipients. UI wage data does not include individuals who are self-employed, employed out of state, employed by the military or federal government, those without a valid social security number, or making less than minimum wage. Sources: State University Database System (SUDS), Florida Education \& Training Placement Information Program (FETPIP), National Student Clearinghouse.

For each of the last four years of data, the annual undergraduate total full expenditures (includes direct and indirect expenditures) were divided by the total fundable student credit hours to create a cost per credit hour for each year. This cost per credit hour was then multiplied by 30 credit hours to derive an average annual cost. The average annual cost for each of the four years was summed to provide an average cost per degree for a baccalaureate degree that requires 120 credit hours. Sources: State University Database System (SUDS), Expenditure Analysis: Report IV.

Six Year FTIC Graduation Rate
This metric is based on the percentage of first-time-in-college (FTIC) students who started in the Fall (or summer continuing to Fall) term and had graduated from the same institution within six years. Source: Accountability Report (Table 4D).
Academic

Progress Rate

2nd Year Retention

with GPA Above 2.0
This metric is based on the percentage of first-time-in-college (FTIC) students who started in the Fall (or summer continuing to Fall) term and were enrolled full-time in their first semester and were still enrolled in the same institution during the Fall term following their first year with had a grade point average (GPA) of at least 2.0 at the end of their first year (Fall, Spring, Summer). Source: Accountability Report (Table 4B).
University Access Rate Percent of Undergraduates with a Pell-grant

\section{Bachelor's Degrees within Programs of Strategic Emphasis}

\section{Graduate Degrees within Programs of Strategic Emphasis}

This metric is based the number of undergraduates, enrolled during the fall term, who received a Pell-grant during the fall term. Unclassified students, who are not eligible for Pellgrants, were excluded from this metric. Source: Accountability Report (Table 3E).

This metric is based on the number of baccalaureate degrees awarded within the programs designated by the Board of Governors as 'Programs of Strategic Emphasis'. A student who has multiple majors in the subset of targeted Classification of Instruction Program codes will be counted twice (i.e., double-majors are included). Source: Accountability Report (Table $4 \mathrm{H})$.

This metric is based on the number of graduate degrees awarded within the programs designated by the Board of Governors as 'Programs of Strategic Emphasis'. A student who has multiple majors in the subset of targeted Classification of Instruction Program codes will be counted twice (i.e., double-majors are included). Source: Accountability Report (Table $5 C)$. 


\section{BOG Choice Metrics}

\section{Percent of Bachelor's Degrees Without Excess Hours}

This metric is based on the percentage of baccalaureate degrees awarded within $110 \%$ of the credit hours required for a degree based on the Board of Governors Academic Program Inventory.

Note: It is important to note that the statutory provisions of the "Excess Hour Surcharge" (1009.286, FS) have been modified several times by the Florida Legislature, resulting in a phased-in approach that has created three different cohorts of students with different requirements. The performance funding metric data is based on the latest statutory requirements that mandates $110 \%$ of required hours as the threshold. In accordance with statute, this metric excludes the following types of student credits (ie, accelerated mechanisms, remedial coursework, non-native credit hours that are not used toward the degree, non-native credit hours from failed, incomplete, withdrawn, or repeated courses, credit hours from internship programs, credit hours up to 10 foreign language credit hours, and credit hours earned in military science courses that are part of the Reserve Officers' Training Corps (ROTC) program). Source: State University Database System (SUDS).

\section{BOT Choice Metrics}

Number of Postdoctoral
Appointees
USF

This metric is based on the number of post-doctoral appointees at the beginning of the academic year. A postdoctoral researcher has recently earned a doctoral (or foreign equivalent) degree and has a temporary paid appointment to focus on specialized research/scholarship under the supervision of a senior scholar.

Source: National Science Foundation/National Institutes of Health annual Survey of Graduate Students and Postdoctorates in Science and Engineering (GSS).

\section{Preeminent Research University Funding Metrics}

\section{Average GPA and SAT Score}

\section{Ranking}

An average weighted grade point average of 4.0 or higher and an average SAT score of 1200 or higher for fall semester incoming freshmen, as reported annually in the admissions data that universities submit to the Board of Governors. This data includes registered FTIC (student type='B','E') with an admission action of admitted or provisionally admitted ('A','P', 'X').

A top-50 ranking on at least two well-known and highly respected national public university rankings, reflecting national preeminence, using most recent rankings, includes: Princeton Review, Fiske Guide, QS World University Ranking, Times Higher Education World University Ranking, Academic Ranking of World University, US News and World Report National University, US News and World Report National Public University, US News and World Report Liberal Arts Colleges, Forbes, Kiplinger, Washington Monthly Liberal Arts Colleges, Washington Monthly National University, and Center for Measuring University Performance. 
Freshman Retention Rate (Full-time, FTIC)
Freshman Retention Rate (Full-time, FTIC) as reported annually to the Integrated Postsecondary Education Data System (IPEDS). The retention rates that are reported in the Board's annual Accountability report are preliminary because they are based on student enrollment in their second fall term as reported by the 28th calendar day following the first day of class. When the Board of Governors reports final retention rates to IPEDS in the Spring (usually the first week of April), that data is based on the student enrollment data as reported after the Fall semester has been completed. The preliminary and final retention rates are nearly identical when rounded to the nearest whole number.

Cohorts are based on undergraduate students who enter the institution in the Fall term (or Summer term and continue into the Fall term). Percent Graduated is based on federal rate and does not include students who originally enroll as part-time students, or who transfer into the institution. This metric complies with the requirements of the federal Student Right to Know Act that requires institutions to report the completion status at $150 \%$ of normal time (or six years). For more information about how this data is calculated, see: http://www.flbog.edu/about/budget/docs/performance_funding/PBF_GRADUATION_and_RETENTIO N_Methodology_FINAL.pdf.

6-year Graduation Rate (Full-time, FTIC)
National Academy Memberships held by faculty as reported by the Center for Measuring University Performance in the Top American Research Universities (TARU) annual report. National Academy
Memberships

Science \& Engineering Research Expenditures (\$M)
Science \& Engineering Research Expenditures, including federal research expenditures as reported annually to the National Science Foundation (NSF).
Non-Medical

Science \& Engineering Research Expenditures (\$M)

National Ranking in S.T.E.M. Research Expenditures

Patents Awarded

(3 calendar years)
Total S\&E research expenditures in non-medical sciences as reported to the NSF. This removes medical sciences funds (9F \& $12 F$ in HERD survey) from the total S\&E amount.

The NSF identifies 8 broad disciplines within Science \& Engineering (Computer Science, Engineering, Environmental Science, Life Science, Mathematical Sciences, Physical Sciences, Psychology, Social Sciences). The rankings by discipline are determined by BOG staff using the NSF WebCaspar database.

Total patents awarded by the United States Patent and Trademark Office (USPTO) for the most recent three calendar year period. Due to a year-lag in published reports, Board of Governors staff query the USPTO database with a query that only counts utility patents:"(AN/"University Name" AND ISD/yyyymmdd->yyyymmdd AND APT/1)".

Doctoral Degrees Awarded Annually

\section{Number of Post-Doctoral Appointees}

Endowment Size (\$M)
Doctoral degrees awarded annually, as reported annually in the Board of Governors Accountability Report.

The number of Postdoctoral Appointees awarded annually, as reported in the TARU annual report. This data is based on National Science Foundation/National Institutes of Health annual Survey of Graduate Students and Postdoctorates in Science and Engineering (GSS).

This data comes from the National Association of College and University Business Officers (NACUBO) and Commonfund Institute's annual report of Market Value of Endowment Assets - which, due to timing, may release the next fiscal year's data after the Board of Governors Accountability report is published. 


\section{Key Performance Indicators \\ Teaching \& Learning Metrics}

Freshmen in Top 10\%

of HS Graduating Class

Professional/Licensure

Exam First-time Pass Rates

Average Time to Degree

Mean Years for FTIC

in $120 \mathrm{hr}$ programs

FTIC Graduation Rates

In 4 years (or less)

Bachelor's Degrees Awarded

Graduate Degrees Awarded

Bachelor's Degrees Awarded To African-American and Hispanic Students

\section{Adult (Aged 25+) \\ Undergraduates Enrolled}

\section{Percent of Undergraduate FTE Enrolled in Online Courses}

Percent of Bachelor's Degrees
in STEM \& Health

Percent of Graduate Degrees in STEM \& Health
Percent of all degree-seeking, first-time, first-year (freshman) students who had high school class rank within the top $10 \%$ of their graduating high school class. As reported by the university to the Common Data Set (C10).

The number of exams with first-time pass rates above and below the national or state average, as reported in the annual Accountability report, including: Nursing, Law, Medicine (3 subtests), Veterinary, Pharmacy, Dental (2 subtests), Physical Therapy, and Occupational Therapy.

This metric is the mean number of years between the start date (using date of most recent admission) and the end date (using the last month in the term degree was granted) for a graduating class of first-time, single-major baccalaureates in 120 credit hour programs within a (Summer, Fall, Spring) year.

As reported in the annual Accountability report (table 4D), First-time-in-college (FTIC) cohort is defined as undergraduates entering in fall term (or summer continuing to fall) with fewer than 12 hours earned since high school graduation. The rate is the percentage of the initial cohort that has either graduated from or is still enrolled in the same institution by the fourth academic year. Both full-time and part-time students are used in the calculation. The initial cohort is revised to remove students, who have allowable exclusions as defined by IPEDS, from the cohort.

This is a count of baccalaureate degrees awarded as reported in the annual Accountability Report (Table 4G).

This is a count of graduate degrees awarded as reported in the Accountability Report (Table $5 B)$.

Non-Hispanic Black and Hispanic do not include students classified as Non-Resident Alien or students with a missing race code - as reported in the Accountability Report (table 4I).

Students who earn two distinct degrees in the same term are counted twice - whether their degrees are from the same six-digit CIP code or different CIP codes. Students who earn only one degree are counted once - even if they completed multiple majors or tracks. Percentage of Degrees is based on the number of baccalaureate degrees awarded to non-Hispanic Black and Hispanic students divided by the total degrees awarded - excluding those awarded to non-resident aliens and unreported.

This metric is based on the age of the student at the time of enrollment (not upon entry). Age acts as a surrogate variable that captures a large, heterogeneous population of adult students who often have family and work responsibilities as well as other life circumstances that can interfere with successful completion of educational objectives.

Full-time Equivalent (FTE) student is a measure of instructional activity that is based on the number of credit hours that students enroll. FTE is based on the US definition, which divides undergraduate credit hours by 30 . Distance Learning is a course in which at least 80 percent of the direct instruction of the course is delivered using some form of technology when the student and instructor are separated by time or space, or both (per 1009.24(17), F.S.).

The percentage of baccalaureate degrees that are classified as STEM by the Board of Governors in the SUS program inventory as reported in the annual Accountability Report (Table 4H).

The percentage of baccalaureate degrees that are classified as STEM by the Board of Governors in the SUS program inventory as reported in the annual Accountability Report (Table 5C). 


\section{Key Performance Indicators (continued) \\ Scholarship, Research \& Innovation Metrics}

Awards include: American Council of Learned Societies (ACLS) Fellows, Beckman Young Investigators, Burroughs Wellcome Fund Career Awards, Cottrell Scholars, Fulbright American Scholars, Getty Scholars in Residence, Guggenheim Fellows, Howard Hughes Medical Institute Investigators, Lasker Medical Research Awards, MacArthur Foundation Fellows, Andrew W. Mellon Foundation Distinguished Achievement Awards, National

Faculty Awards Endowment for the Humanities (NEH) Fellows, National Humanities Center Fellows, National Institutes of Health (NIH) MERIT, National Medal of Science and National Medal of Technology, NSF CAREER awards (excluding those who are also PECASE winners), Newberry Library Long-term Fellows, Pew Scholars in Biomedicine, Presidential Early Career Awards for Scientists and Engineers (PECASE), Robert Wood Johnson Policy Fellows, Searle Scholars, Sloan Research Fellows, Woodrow Wilson Fellows. As reported by the Top American Research Universities - see: http://mup.asu.edu/research data.html.

Total Research Expenditures (\$M)

Percent of R\&D Expenditures funded from External Sources

Licenses/Options Executed
Total expenditures for all research activities (including non-science and engineering activities) as reported in the National Science Foundation annual survey of Higher Education Research and Development (HERD).

This metric reports the amount of research expenditures that was funded from federal, private industry and other (non-state and non-institutional) sources.

Source: National Science Foundation annual survey of Higher Education Research and Development (HERD).

Licenses/options executed in the fiscal year for all technologies as reported in the annual Accountability Report (table 6A).

Number of Start-up Companies

The number of start-up companies that were dependent upon the licensing of University technology for initiation as reported in the annual Accountability Report (table 6A). 


\section{Student Debt Summary}

\section{Percent of Bachelor's Recipients with Debt}

This is the percentage of bachelor's graduates in a given academic year who entered the university as a first-time-in-college (FTIC) student and who borrowed through any loan programs (institutional, state, Federal Perkins, Federal Stafford Subsidized and unsubsidized, private) that were certified by your institution - excludes parent loans. Source: Common Dataset (H4).

This is the average amount of cumulative principal borrowed (from any loan program certified by the institution) for each native, FTIC bachelor's recipient in a given academic year that graduated with debt - see metric definition above. This average does NOT include students who did not enter a loan program that was certified by the institution. Source: Common Dataset (H5).

Student loan cohort default rate (CDR) data includes undergraduate and graduate students, and refers to the three federal fiscal year period when the borrower enters repayment and ends on the second fiscal year following the fiscal year in which the borrower entered repayment. Cohort default rates are based on the number of borrowers who enter repayment, not the number and type of loans that enter repayment. A borrower with multiple loans from the same school whose loans enter repayment during the same cohort fiscal year will be included in the formula only once for that cohort fiscal year. Default rate debt includes: Federal Stafford Loans, and Direct Stafford/Ford Loans - for more information see: http://ifap.ed.gov/DefaultManagement/CDRGuideMaster.html.

\begin{tabular}{|c|c|c|c|}
\hline $\begin{array}{l}\text { Cohort } \\
\text { Fiscal } \\
\text { Year }\end{array}$ & $\begin{array}{c}\text { Year } \\
\text { Published }\end{array}$ & $\begin{array}{l}\text { Borrowers in the Numerator } \\
\text { Borrowers in the Denominator }\end{array}$ & $\begin{array}{l}\frac{\text { 3-Yr Time Period }}{\text { (Numerator) }} \\
\text { 1-Yr Time Period } \\
\text { (Denominator) }\end{array}$ \\
\hline 2009 & 2012 & $\begin{array}{l}\text { Borrowers who entered repayment in } 2009 \\
\text { and defaulted in 2009, } 2010 \text { or } 2011 \\
\text { Borrowers who entered repayment in } 2009\end{array}$ & $\frac{10 / 01 / 2008 \text { to } 9 / 30 / 2011}{10 / 01 / 2008 \text { to } 9 / 30 / 2009}$ \\
\hline 2010 & 2013 & $\begin{array}{l}\text { Borrowers who entered repayment in } 2010 \\
\text { and defaulted in } 2010,2011 \text { or } 2012 \\
\text { Borrowers who entered repayment in } 2010\end{array}$ & $\frac{10 / 01 / 2009 \text { to } 9 / 30 / 2012}{10 / 01 / 2009 \text { to } 9 / 30 / 2010}$ \\
\hline 2011 & $2014^{*}$ & $\begin{array}{l}\text { Borrowers who entered repayment in } 2011 \\
\text { and defaulted in } 2011,2012 \text { or } 2013 \\
\text { Borrowers who entered repayment in } 2011\end{array}$ & $\frac{10 / 01 / 2010 \text { to } 9 / 30 / 2013}{10 / 01 / 2010 \text { to } 9 / 30 / 2011}$ \\
\hline 2012 & 2015 & $\begin{array}{l}\text { Borrowers who entered repayment in } 2012 \\
\text { and defaulted in } 2012,2013 \text { or } 2014 \\
\text { Borrowers who entered repayment in } 2012\end{array}$ & $\frac{10 / 01 / 2011 \text { to } 9 / 30 / 2014}{10 / 01 / 2011 \text { to } 9 / 30 / 2012}$ \\
\hline 2013 & 2016 & $\begin{array}{l}\text { Borrowers who entered repayment in } 2013 \\
\text { and defaulted in 2013,2014 or } 2015 \\
\text { Borrowers who entered repayment in } 2013\end{array}$ & $\frac{10 / 01 / 2012 \text { to } 9 / 30 / 2015}{10 / 01 / 2012 \text { to } 9 / 30 / 2013}$ \\
\hline 2014 & 2017 & $\begin{array}{l}\text { Borrowers who entered repayment in } 2014 \\
\text { and defaulted in } 2014,2015 \text { or } 2016 \\
\text { Borrowers who entered repayment in } 2014\end{array}$ & $\frac{10 / 01 / 2013 \text { to } 9 / 30 / 2016}{10 / 01 / 2013 \text { to } 9 / 30 / 2014}$ \\
\hline 2015 & 2018 & $\begin{array}{l}\text { Borrowers who entered repayment in } 2015 \\
\text { and defaulted in } 2015,2016 \text { or } 2017 \\
\text { Borrowers who entered repayment in } 2015\end{array}$ & $\frac{10 / 01 / 2014 \text { to } 9 / 30 / 2017}{10 / 01 / 2014 \text { to } 9 / 30 / 2015}$ \\
\hline
\end{tabular}

